\section{Rubicon Model of Action Phases}

To differentiate and integrate both the selection and realization of goals, the Rubicon model of action phases was developed. The model describes successful goal pursuit as solving four consecutive tasks: choosing between potential goals, planning the implementation of a chosen goal, acting on the chosen goal, and assessing what has been achieved by acting on the goal and what still needs to be achieved by further acting on the goal. Thus, the Rubicon model of action phases posits four distinct phases of goal pursuit: (1) the predecisional phase, in which the pros and cons of one's wishes and desires are deliberated by assessing the desirability of expected outcomes and the question of feasibility (i.e., Can I obtain the desired outcomes if I wanted to?); (2) the postdecisional phase, in which the implementation of the chosen goal is planned by deciding on when, where, and how one wants to act toward the goal; (3) the actional phase, in which one progresses toward the goal by initiating goal-directed behaviors and bringing them to a successful ending; (4) finally, the postactional phase in which the achieved outcomes of the goal-directed behavior are evaluated by looking backward (i.e., How successfully did I perform the goal-directed behavior?) and forward (i.e., What needs to be done still to achieve the desired outcomes implied by my goal?).

These four phases are separated by three clear transition points: (1) deciding to strive for the realization of certain wishes and desires, thus transforming them into goals (at the end of the predecisional phase); (2) the initiation of actions suited to attain these goals (at the end of the preactional phase); and (3) the evaluation of the achieved outcomes of these goal-directed actions (at the end of the actional phase). The transition point at the end of the first phase is called the transition of the Rubicon. This metaphor comes from Julius Caesar's crossing of the northern Italian Rubicon River with his army after some hesitations in 49 в.C.E., thereby initiating a civil war. By crossing the Rubicon, Caesar committed himself to conquer or to perish. Thus, the metaphor "crossing the Rubicon" symbolizes that as soon as one has decided to pursue a select wish or desire, the pro versus con deliberation is terminated, and one is strongly committed to act. Thus, at the end of the predecisional phase, the deliberation is replaced by a sense of determination to actually realize the former wish or desire that is now experienced as a firm goal.

Different modes of thought are associated with each of the four action phases-the so-called action mindsets. By getting involved with the distinct tasks posed in each of the four phases, certain ways of thinking become more prominent (i.e., unique cognitive procedures are activated). The deliberative mind-set is associated with the predecisional phase. It emerges when people start to think about an unresolved personal problem that is still a wish or desire, thinking of the short-term and long-term pros and cons of both making and not making the decision to realize it. The implemental mind-set is associated with the postdecisional phase. It originates when people start to plan the steps they want to take to actually realize a chosen goal. These plans specify when, where, and how one intends to execute each of these steps.

To investigate the cognitive, self-evaluative, and behavioral consequences of the deliberative and implemental mind-sets, the following experimental paradigm was invented. Research participants are made to believe that they have to perform two different, subsequent experiments (usually performed by two different experimenters). The first experimenter then induces the deliberative and the implemental mind-sets. The deliberative mind-set is induced by having participants deliberate a still unresolved personal problem (e.g., 
Should I move to a different apartment?). The implemental mind-set is induced by having participants plan the implementation of a chosen project to be resolved in the near future (e.g., moving into a different apartment). The second experimenter, being blind to this manipulation of mind-sets, then asks participants to perform certain tasks or answer questionnaires that tap into the hypothesized cognitive features of the two mind-sets. Numerous studies in social and motivation psychology have used this paradigm showing that the deliberative and the implemental mind-sets thus created have distinct consequences.

People in a deliberative mind-set usually show the following attributes: (a) They evaluate their selves accurately (i.e., rate themselves realistically with regard to intelligence, attractiveness, etc.), (b) they show reduced positive illusions of control over frequent outcomes that are uncontrollable, (c) they make less positive illusionary judgments of their invulnerability to controllable (e.g., divorce, having a drinking problem) and uncontrollable risks (e.g., death of a loved one), (d) they are impartial in the sense that they appraise desirability-related information even-handedly, (e) they are particularly effective in processing desirability-related information, and (f) they are openminded as their processing of incidental information is generally very effective.

In contrast, people in an implemental mind-set show quite different attributes: (a) They evaluate themselves in a very positive illusionary manner (e.g., they rate themselves as much more intelligent and attractive than the average person in their peer group), (b) they show strong illusions of control over frequent, but uncontrollable outcomes, (c) they make very positive illusionary judgments of their vulnerability to controllable and uncontrollable outcomes, (d) they are partial in the sense that they focus on positive desirability-related information more than on negative desirability-related information, (e) they are particularly effective in processing information related to the realization of goals, and (f) they are closed-minded in the sense that they are rather sluggish in processing incidental information.

Recently, these effects of implemental mind-sets (enhanced self-efficacy, optimistic outcome expectations, perceptions of the task at hand as easy, etc.) were shown help people to succeed in their ongoing goal pursuits. Furthermore, people in an implemental mindset are more optimistic in their forecasts of the survival of their romantic relationships than deliberative mind-set individuals. When choosing test materials of different difficulty, people in implemental mind-sets preferred more difficult tasks than did people in deliberative mind-sets. Moreover, those in implemental mind-sets overestimated their probability of success as compared with people in deliberative mind-sets.

Finally, there are individual differences in the ability of activating deliberative and implemental mind-sets and their effects on cognition and behavior. Deliberative and implemental mind-set effects are moderated by a person's level of achievement motivation, social anxiety, and goal commitment. For instance, people whose achievement motive is strongly determined by hope for success (in contrast to fear of failure) show a strong illusionary optimism when they are in an implemental mind-set compared with a deliberative mind-set. Conversely, fear of failure people when in a deliberative mind-set boost their self-perception of competence (i.e., show illusionary optimism), but not when they are in an implemental mind-set.

The Rubicon model of action phases with its associated mind-set theory has stimulated a re-conceptualization of the classic concept of motivation. In the past, the term motivation referred to both the readiness to choose a certain course of action and the intensity and effectiveness with which the chosen course of action was implemented. Nowadays, one discusses only issues of choosing a course of action in motivational terms by pointing to the motivational variables of desirability and feasibility. However, the issue of successful implementation of a chosen course of action is considered to be volitional in nature. That is, it depends on people's willpower and their possession of relevant self-regulation skills whether a chosen course of action is ultimately implemented. The mind-set theory associated with the Rubicon model turned out to be conceptually very influential too. Theories of action control that distinguish between types of goals (e.g., abstract vs. concrete, promotion vs. prevention, learning vs. performance) have tried to test their hypotheses by creating respective mind-sets-for instance, creating why versus how mind-sets to assess differences between the pursuit of goals construed at a high versus low level of abstraction.

Anja Achtziger

Peter M. Gollwitzer 